\title{
BMJ Open ACCESS HD pilot: A randomised feasibility trial Comparing Catheters with fistulas in Elderly patientS Starting haemodialysis
}

\author{
Robert Quinn, ${ }^{1,2}$ Pietro Ravani, ${ }^{1,2}$ The ACCESS HD Investigators
}

To cite: Quinn R, Ravani P, The ACCESS HD Investigators. ACCESS HD pilot: A randomised feasibility trial Comparing Catheters with fistulas in Elderly patientS Starting haemodialysis. BMJ Open 2016;6:e013081.

doi:10.1136/bmjopen-2016013081

- Prepublication history and additional material is available. To view please visit the journal (http://dx.doi.org/ 10.1136/bmjopen-2016013081)

Received 17 June 2016 Revised 18 October 2016 Accepted 27 October 2016

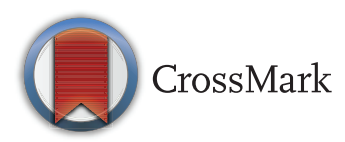

${ }^{1}$ Cumming School of Medicine/Libin Cardiovascular Institute, University of Calgary, Calgary, Alberta, Canada

${ }^{2}$ Cumming School of Medicine/O'Brien Institute of Public Health, University of Calgary, Calgary, Alberta, Canada

\section{Correspondence to}

Dr Rob Quinn;

Rob.Quinn@ahs.ca and

Dr Pietro Ravani; pravani@ucalgary.ca

\section{ABSTRACT}

Introduction: The selection of the type of vascular access for haemodialysis is an important intervention question. However, only observational studies are available to inform decision-making in this area, and they are at high risk of selection bias. While a clinical trial comparing the effects of the 2 most frequently chosen strategies for haemodialysis access (fistulas and catheters) on patient important and 'hard' clinical end points is needed, the feasibility of such a trial is uncertain.

Methods and analysis: This open-label pilot randomised controlled trial will test the feasibility and safety of randomising elderly people ( $\geq 65$ years) who start haemodialysis with a central venous catheter (the most common initial type of haemodialysis access), and are eligible to receive a fistula, to a catheter-based strategy (comparator) or to a fistula-based strategy (intervention). We will enrol 100 patients at 10 centres across Canada. Participants assigned to the catheterstrategy arm will continue to use catheters; participants assigned to the fistula-strategy arm will receive a surgical attempt at fistula creation. The inclusion criteria are designed to minimise the risk of protocol violation and attrition. The primary outcome is feasibility, which we will assess by measuring: (1) the proportion of participants deemed eligible for the trial who consent to randomisation; and (2) the proportion of participants randomised to the intervention who receive the fistula surgery within 90 days of randomisation. Secondary outcomes will include safety outcomes, the reasons people and healthcare providers may not accept randomisation, and the reasons sites may not adhere to the trial protocol.

Ethics and dissemination: The Conjoint Health Research Ethics Board at the University of Calgary approved the study protocol. We will submit the results of this feasibility study in a peer-reviewed journal.

Trial registration number: NCT02675569,

Pre-results.

\section{BACKGROUND}

Worldwide, over 2 million people receive haemodialysis therapy for end-stage kidney

\section{Strengths and limitations of this study}

- This feasibility study is the first effort to prove that randomising patients to different access strategies is feasible and safe, which is a vital first step to conducting the first randomised controlled trial in this area.

- This feasibility study has the same design as the hard outcome trial (including the data collection for the primary and secondary outcomes of the hard outcome trial); if the pilot is successful participants will be rolled from the pilot into the larger trial.

- The questions addressed in this pilot and the hard outcome trial that will follow if the pilot is feasible have been ranked among the top 10 research priorities in dialysis by patients and healthcare providers.

- The question is asked in people over 65 years of age; results will need to be interpreted and generalised accordingly; the question is asked following haemodialysis start with a catheter (as opposed to the predialysis setting); predialysis education about vascular access may affect the feasibility of this trial.

- Owing to the nature of the intervention, the study is blinded for the outcome assessors only.

failure. ${ }^{1-3}$ These people require access to the bloodstream by means of a fistula or a catheter (the most common forms of vascular access) to connect to the blood purification machine. Guidelines recommend a fistula for haemodialysis access based on evidence from large observational studies showing that fistula use is associated with better clinical outcomes (including mortality) and lower costs than catheter use. ${ }^{45}$ However, there has never been a randomised controlled trial (RCT) comparing fistulas with catheters, and existing studies addressing this question are at high risk of bias, especially selection bias. ${ }^{6-10}$ The choice of haemodialysis access is a common intervention question in clinical 
practice and is very relevant for patients and health policymakers because it impacts morbidity, mortality, quality of life and health costs. ${ }^{11-13}$

In Canada, more than $80 \%$ of people with kidney failure who start haemodialysis use a catheter for vascular access, and are faced with the decision to either continue with a catheter or attempt fistula creation. ${ }^{14} 15$ Although fistula creation is recommended regardless of age, ${ }^{16}$ promoting fistulas in the elderly may be ineffective as in this patient population fistulas are less likely to become suitable for haemodialysis than in younger people. ${ }^{17}$ In addition, even when fistula attempts are successful, elderly people may not receive the same benefit as younger people due to their shorter life expectancy. Finally, recent data suggest that the risks associated with catheters (eg, catheter-related bacteraemia) may be lower in the elderly ${ }^{18}$ and that a person's preference for the type of vascular access varies by age, with the elderly being more likely to prefer catheters to fistulas. ${ }^{19}$ For these reasons, the expected benefits of fistula creation are even more uncertain in the elderly.

A large RCT to determine the benefits and harms of one form of vascular over the other may not be feasible for several reasons. First, people may develop strong preferences for a particular form of vascular access based on the information presented during education about their options. Fistulas require a surgical intervention that can disfigure the arm and require the insertion of needles for dialysis at each treatment (ie, three times per week). Catheters impose lifestyle restrictions (eg, avoidance of swimming) and are visible over the chest. As a consequence, patients may develop strong preferences and be less willing to consent to randomisation. Second, physicians may not be willing to enrol people into a trial due to guideline recommendations and/or existing incentives to increase the number of people treated with fistulas. Third, programmes may not be able to arrange for a fistula creation within a reasonable time (eg, 90 days from randomisation), thereby increasing the risk that consent is withdrawn before the fistula is created or longer exposure to catheters may outdo potential benefits of fistulas. These considerations motivated the design of the present pilot RCT to test the feasibility of a larger, hard outcome trial.

\section{METHODS/DESIGN}

Objective and outcomes

This pilot RCT will test the feasibility and safety of randomising elderly people who start haemodialysis with a catheter to continued use of a catheter or attempt at fistula creation.

\section{Design}

This feasibility RCT will be multicentre, parallel-arm and open-label in design. The report of the study protocol conforms to the Standard Protocol Items: Recommendations for Interventional Trials (see online
Supplementary material file). ${ }^{20}$ The study process is illustrated in figure 1 . We will randomise 100 elderly people (age $\geq 65$ ) with end-stage kidney failure starting haemodialysis with a catheter in 10 Canadian haemodialysis programmes (1:1) to continued use of catheters (comparator) or receive an attempt at fistula creation (intervention). The pilot RCT will consist of 6 months of site preparation, $\sim 24$ months of participant accrual and 6 months of additional follow-up time for the last randomised participant. An additional 6 months will be used to assess the preliminary data, prepare the report of this pilot RCT and to prepare and submit funding applications for a larger hard outcome RCT. Participants in the pilot RCT will be rolled into the hard outcome RCT, and will be followed for a total of 36 months (3 years) after randomisation (box 1).

\section{Preliminary data}

Historical data collected as part of quality improvement efforts ${ }^{21}$ indicate that $\sim 500$ new people start dialysis each year at four of the participating sites (Southern Alberta Renal Program, Calgary; The Ottawa Hospital, Ottawa; St Joseph's Health Centre, Hamilton; Sunnybrook Health Sciences Centre, Toronto). Of these people, $\sim 80 \%$ start on haemodialysis $(\mathrm{N}=400)$ and $80 \%$ of those people use a catheter for haemodialysis access $(\mathrm{N}=320)$. According to the same data, at least $50 \%(\mathrm{~N}=160)$ meet the inclusion criteria and if we conservatively assume that $25 \%$ of them will consent to randomisation, at least 80 participants will be recruited over 2 years across these four sites. An additional 20 participants will be recruited among the other six participating Canadian centres. The 100-person sample size is reasonable to address the feasibility question of the pilot RCT (ie, whether randomising people to different access strategies is possible). However, identifying barriers to enrolment and thereby, understanding how to maximise enrolment rate at all participating centres will be critical to maximise recruitment rate in the hard outcome RCT. We will continue to monitor these feasibility indicators as the pilot ACCESS HD transitions into the hard outcome ACCESS HD trial.

\section{Participants}

We plan to enrol 100 people in total across 10 centres in Canada. This will be possible within 2 years if we enrol $25 \%$ of eligible people. We will screen all people who start haemodialysis with a catheter for inclusion in our trial, including people who start haemodialysis in hospital and those who start haemodialysis urgently. We will assess eligibility of these people once they are sufficiently stable to provide informed consent (see the Eligibility criteria section). We will also include people who start as outpatients and those previously treated with haemodialysis therapy before they changed modality to peritoneal dialysis or transplantation. However, only participants with one previous unsuccessful fistula attempt (randomisation will be stratified on this variable) will be eligible. In each centre, we have 


\section{Enrollment}

Assessed for eligibility

Excluded

- Not meeting inclusion criteria

- Declined to participate

- Other reasons

PILOT Analysis $1=$ At least $25 \%$ of all

eligible expected to be randomized

Randomized

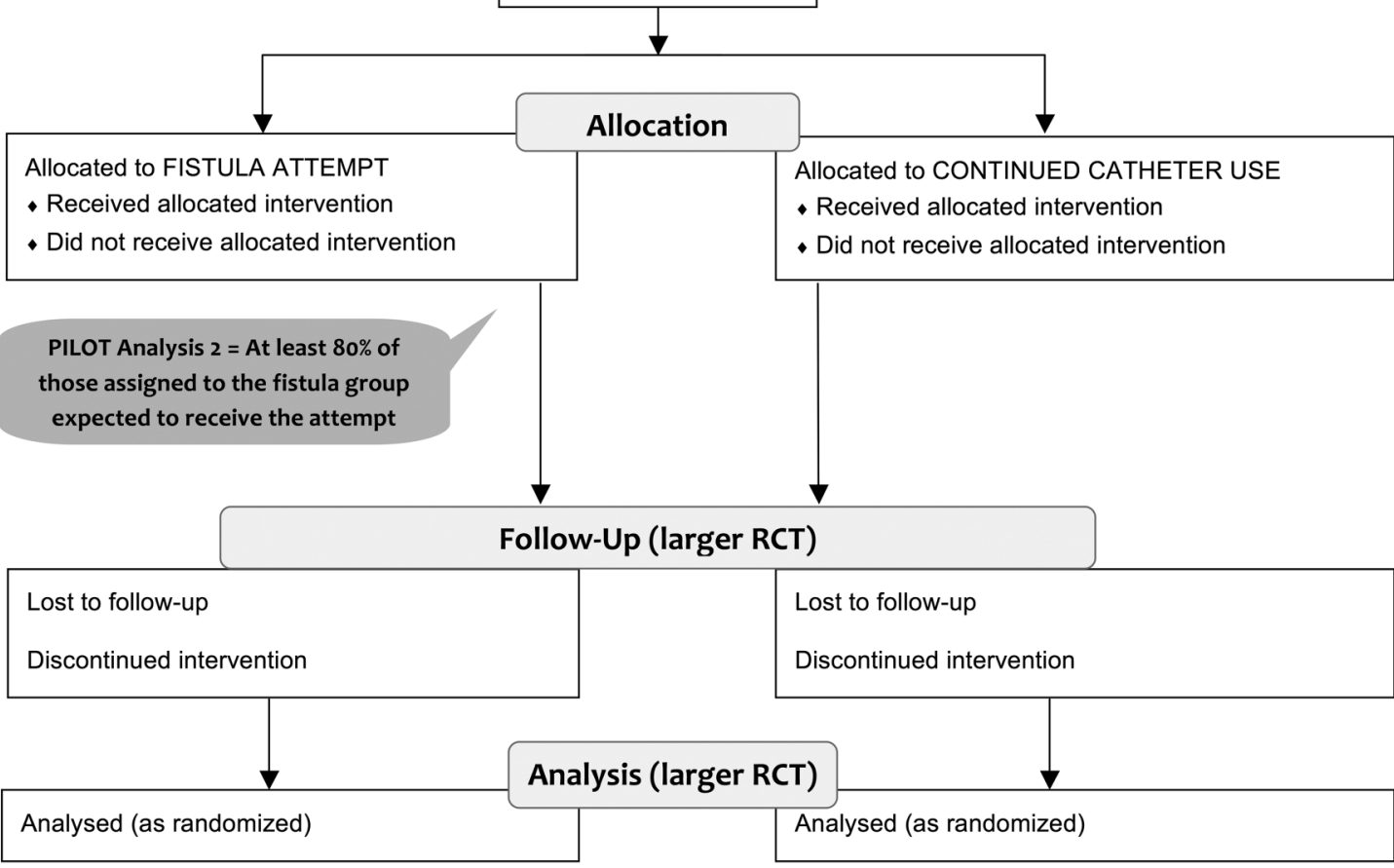

Figure 1 Flow diagram.

implemented strategies to identify all new haemodialysis starts within the programme and ensure adequate enrolment to reach the target sample size. We will assess eligibility of all people starting haemodialysis with a catheter and track the time of this assessment relative to the dialysis start date.

\section{Box 1 Research question (PICOT format)}

Question: Is it feasible to randomly allocate people over 65 who start haemodialysis with a catheter to either continued use of catheter or attempt fistula creation?

Population: people over the age of 65 who started haemodialysis with a catheter and are eligible for a fistula

Intervention: attempt at fistula creation

Comparator. continue with the use of catheters for haemodialysis Outcomes: feasibility (randomisation of at least 1 in 4 eligible participants; fistula attempted within 3 months of allocation in at least 3 out of 4 participants assigned to the intervention arm) Time: Consent must be obtained within 4 months of haemodialysis start; randomisation must occur within 14 days of attaining participant consent; participants are followed for 6 months since randomisation in this pilot study and then rolled into the hard outcome trial.

\section{Adherence and attrition}

One of the objectives of this feasibility RCT is to measure adherence (ie, arranging for a fistula creation within 90 days of randomisation in those assigned to the fistula arm) to demonstrate that centres will adhere to the trial protocol. We will monitor compliance with follow-up visits and the completion of trial questionnaires. We currently track all incident haemodialysis patients at several Canadian sites using a high-quality, web-based platform for the purpose of quality improvement (Dialysis Measurement, Analysis and Reporting (DMAR) System). ${ }^{21}$ Analysis of two representative programmes suggests that $5 \%$ of people starting dialysis transfer out of a dialysis programme or are lost to follow-up over the first year of dialysis.

\section{Eligibility criteria}

Table 1 summarises inclusion and exclusion criteria. There are no well-accepted criteria to determine eligibility for a fistula attempt, ${ }^{22}$ so we will take a pragmatic approach. The team will be free to order any additional investigations required to determine eligibility (eg, vascular mapping), as per local practice. We will collect detailed data around the assessment process at each site 
Table 1 Eligibility criteria

\section{Inclusion criteria}

1. Adult patients age $\geq 65$

2. Started haemodialysis using a tunnelled, or non-tunnelled catheter for vascular access

3. Treated with dialysis for 120 days or less at the time of consent (134 days or less at the time of randomisation) 4. Clinically stable as assessed by the treating nephrologist (able to provide consent within 120 days of haemodialysis start)

5. Haemodialysis is the intended long-term modality of treatment

6. End-stage (permanent) kidney failure unlikely to recover kidney function according to the attending nephrologist

7. Eligible for a fistula attempt as determined by the local multidisciplinary access team

8. Planning to remain in the current dialysis centre for at least 6 months

\section{Exclusion criteria}

1. Started haemodialysis with a fistula or have a patent fistula already in place

2. More than one unsuccessful fistula attempt prior to starting dialysis

3. Has had a prior arteriovenous graft creation

4. Imminent transplant planned (within 6 months, must be booked)

5. Metastatic malignancies or other condition associated with a life expectancy of $<6$ months, in the opinion of the attending nephrologist during assessment of site feasibility. The determination of eligibility will be the consensus opinion of the primary nephrologist, access surgeon and access nurse (more often based on a series of independent assessments). Only after all assessments and required investigations are completed, will participants be eligible for randomisation.

\section{Participant identification and randomisation \\ Participant identification}

The trial coordinator at each site will track people who start haemodialysis and are 65 years of age or older. The coordinator will contact the primary nephrologist of potentially eligible participants to introduce the topic of the trial and advise the patient that the trial team will approach them to discuss participation in the trial. After written informed consent is obtained, the trial coordinator will confirm trial eligibility. All screened people and reasons for exclusion will be documented in a screening $\log$ and a minimal data set in the electronic case report form (eCRF) will be collected for all eligible patients including those who do not consent.

\section{Randomisation and blinding}

Participants will be allocated equally (1:1) to either a fistula attempt (intervention) or continued use of a tunnelled catheter (comparator), stratified by centre and whether or not they have had a prior, unsuccessful fistula attempt. Stratification by these variables will protect randomisation in case the eligibility criteria change for the hard outcome RCT. An independent statistician at the coordinating centre will generate the allocation sequences and keep them concealed from the clinical investigators. Each randomisation list will be generated using randomly permuted blocks of varying sizes. Since this is an open-label trial, knowledge of block sizes could, under certain situations, permit determination of future allocations. For this reason, block size will remain concealed throughout the trial. Randomisation will be operationalised using a web-based, central system (Medidata RAVE). After confirming participant eligibility and obtaining signed consent, the local trial coordinator will obtain the participant's assignment electronically via Medidata RAVE. We will not blind participants or providers because the creation of fistulas requires a surgical procedure in the arm, results in clearly visible changes to the appearance of the arm and requires needling of the access for dialysis (as opposed to hub connections). However, outcome adjudicators will be blinded for all outcomes of the larger RCT.

\section{Trial treatments and cointerventions}

\section{Intervention arm}

Participants will undergo an attempt at fistula creation by an experienced surgeon. Preoperative studies (angiography and/or ultrasound mapping) will be allowed as dictated by indications, local practice and physician preferences. We will collect information about policies and practices, including, for example, the approach to selection of the location of the anastomosis from distal locations (radio-cephalic fistula first) to more proximal locations (brachio-cephalic fistula next and brachiobasilic anastomosis as last choice). Variants on these types of fistulas as well as other options for fistula creation will be at the discretion of the local vascular access team, as per usual practice. The protocol requires that the first fistula attempt occurs within 90 days of randomisation and this pilot RCT will measure adherence to this requirement as a measure of feasibility. In the event that the fistula attempt fails or a functioning fistula experiences a complication that renders it unusable for haemodialysis, further fistula attempts will be at the discretion 
of the vascular access team and participant; the participant will remain in the intervention arm and 'analysed as randomised' in the definite trial (intention-to-treat).

\section{Comparator arm}

Participants randomised to the comparator arm will continue to use a tunnelled, central venous haemodialysis catheter for vascular access. Participant using a temporary, non-tunnelled catheter at the time of randomisation will undergo insertion of a tunnelled catheter as per local practice. In the event that the catheter fails or a complication occurs that renders it unusable for haemodialysis, further catheter procedures or fistula attempts will be at the discretion of the vascular access team and participant; the participant will remain in the comparator arm and 'analysed as randomised' in the definite trial (intention-to-treat).

\section{Cotreatments and access monitoring}

All other aspects of general dialysis care will be dictated by local practice and will not be influenced by the trial protocol.

\section{Outcomes}

The primary outcome of this pilot RCT is feasibility. There will be two main determinants of feasibility: our ability to recruit participants and protocol adherence. We will determine the proportion of people meeting our inclusion and exclusion criteria who ultimately consent to randomisation. We aim to consent $\geq 25 \%$ of people who are eligible to be randomised. We will measure protocol adherence based on the ability of the sites to arrange a fistula attempt within 90 days of randomisation to the intervention arm (fistula strategy). We aim to attain a fistula attempt within 90 days of randomisation in $\geq 80 \%$ of participants randomised to the fistula strategy. Other outcomes are reported in box 2 .

\section{Data collection (eCRF)}

We will use Medidata RAVE web-based electronic data capture system for randomisation and electronic data capture.

\section{Trial oversight}

The Applied Health Research Centre (AHRC) at St Michael's Hospital (Toronto, Canada) will act as the trial coordination and data management centre. Trial coordinators or investigators at each centre will determine eligibility, obtain consent for participation in the trial and utilise the online randomisation tool to allocate participants to the appropriate trial intervention. Trial coordinators will complete the web-based eCRF for each participant. All data will be stored on secure internal servers at St Michael's Hospital in Toronto, Ontario. The principal investigators ( $R Q$ and $\mathrm{PR}$ ), in collaboration with the AHRC, will be responsible for trial coordination. The steering committee will include Drs RQ PR, Barrett, Thorpe, Hiremath, Oliver and Walsh, and will

\section{Box 2 Other outcomes}

1. Feasibility-related outcomes: reasons for exclusion or delayed surgery

a. Exclusion of screened people (including competing studies)

b. People declining to participate

c. People considered ineligible for a fistula attempt

d. Delayed access to fistula surgery ( $>90$ days from randomisation)

2. Safety outcomes (to be analysed in the hard outcome RCT)

a. Expected procedure-related events (table 2)

b. Hospital admissions or prolongation of hospitalisation

c. Death potentially related to the trial procedures

3. Other data (to be analysed in the hard outcome RCT)

a. Number and reasons for drop-ins (protocol violations)

b. Number and reasons for drop-outs (protocol violations)

c. Patient-reported outcome measures (PROMs, Quality of life and access questionnaire)

d. Physical function (grip strength)

e. Fistula data (for intervention arm)

f. Resource use

meet before enrolment begins, conduct monthly teleconferences with the AHRC project manager to discuss enrolment rates and operational issues, and again at the end of the trial. The trial protocol received institutional research ethics board (REB) approval in Calgary from the Conjoint Health Research Ethics Board. Approval is currently being sought at all participating sites. All screened people who meet the eligibility criteria will have demographics and clinical characteristics collected in a minimal data set within Medidata RAVE. Every consented participant will have baseline and follow-up data collected as summarised in figure 2 and in the protocol (see online Supplementary material file). Follow-up will start at the time of randomisation in both arms and will continue for 36 months, as participants from this pilot RCT will roll into the hard outcome RCT.

\section{Data safety and monitoring board and end point adjudication committee}

An independent Data Safety and Monitoring Board (DSMB) was formed for the hard outcome RCT. The DSMB will not review the pilot data, but will be responsible for periodic evaluation of the clinical trial data to ensure continued trial participant safety, as well as the scientific validity of the trial. The DSMB met prior to finalising the pilot RCT protocol to draft a functional DSMB charter in preparation for the large RCT. All incidents of participant death that occur during the trial period will be reported.

\section{Protocol violations}

Protocol violations are reported in box 3 .

\section{Statistical considerations}

Power and sample size

The sample size for the feasibility RCT is 100 participants. This number is based on an estimated $25 \%$ 
Table 2 Expected procedure-related events

\section{Fistula strategy}

Allergic reaction (to local anaesthetic)

Allergic reaction (to general anaesthetic)

Bleeding from access site requiring intervention

Wound infection in the fistula

Infection in the bloodstream

Excessive collateral formation on upper arm and chest likely from central

vein stenosis

Aneurysm requiring intervention

\section{Catheter strategy}

Allergic reaction (to local anaesthetic)

Allergic reaction (to general anaesthetic)

Bleeding from access site requiring intervention

Fibrin sheath formation/use of rTPA

Infection (exit site)

Infection (tunnel)

Infection (bloodstream)

Arterial puncture

Myocardial perforation

Arrhythmias
Ischaemia (to nerves or distal extremity) or steal

Syndrome requiring intervention

Heart failure (high output congestive)

Nerve injury

Pulmonary embolism

Excessive arm swelling likely from central venous stenosis acceptance rate and a total of 1000 people expected to start haemodialysis in the participating centres over 2 years. Of these, $80 \%$ will start with a catheter and $50 \%$ are expected to meet the inclusion criteria (conservative number of potentially eligible people $\mathrm{N}=400$ ). The primary indicators of feasibility will be the proportion of people who are randomised among those who are eligible, and the proportion of fistula attempts that occur within 90 days of randomisation. The pilot RCT will support feasibility if these proportions are at least $25 \%$ $(100 / 400$ total people) and $80 \%$ (40/50 participants assigned to the intervention arm), respectively. Considering the small size of the feasibility RCT and the resulting uncertainty around each estimate (eg, for the second estimate $95 \%$ confidence limits range from $9 \%$ to $97 \%$ ), we will continue to monitor feasibility end points after the completion of the pilot RCT and until 100 participants are assigned to the fistula arm in the hard outcome RCT.

\section{Analysis plan}

The primary analysis will be descriptive. The proportion of people meeting each of the feasibility end points (proportion of people meeting inclusion/exclusion criteria who consent to randomisation; proportion of participants randomised to fistula arm who attempt a fistula
Figure 2 Schedule of enrolment, interventions and assessment.

\begin{tabular}{|c|c|c|c|c|c|c|c|c|}
\hline & \multicolumn{8}{|c|}{ STUDY PERIOD } \\
\hline \multirow{2}{*}{$\begin{array}{r}\text { TIMEPOINT } \\
\text { Eligibility screen }\end{array}$} & \multirow{2}{*}{$\frac{\text { Enrolment }}{\mathrm{x}}$} & \multirow[t]{2}{*}{ Allocation } & \multicolumn{5}{|c|}{ Post-allocation (definitive RCT) } & \multirow[t]{2}{*}{$\begin{array}{l}\text { Hard outcomes } \\
\text { (definitive RCT) }\end{array}$} \\
\hline & & & & & & & & \\
\hline \multirow{2}{*}{$\begin{array}{r}\text { Informed consent } \\
\text { Allocation }\end{array}$} & $x$ & & & & & & & \\
\hline & & $x$ & & & & & & \\
\hline \multicolumn{9}{|l|}{ INTERVENTIONS: } \\
\hline \multicolumn{9}{|l|}{ Fistula strategy } \\
\hline \multirow{2}{*}{\multicolumn{9}{|c|}{ Catheter strategy }} \\
\hline & & & & & & & & \\
\hline \multicolumn{9}{|l|}{ ASSESSMENTS: } \\
\hline \multirow{2}{*}{$\begin{array}{r}\text { Demographics } \\
\text { Clinical } \\
\text { characteristics }\end{array}$} & $x$ & & & & & & & \\
\hline & $x$ & & & & & & & \\
\hline Other data & $x$ & & $x$ & $x$ & $x$ & $x$ & etc. & $\mathrm{x}$ \\
\hline OUTCOMES & $\begin{array}{c}\text { PILOT } \\
\text { (feasibility) }\end{array}$ & $\begin{array}{c}\text { PILOT } \\
\text { (feasibility) }\end{array}$ & & & & & & Larger RCT \\
\hline
\end{tabular}




\section{Box 3 Protocol violations}

1. Failure to attempt the fistula within 90 days of randomisation

2. Drop-ins (for reasons of safety, or as requested by the participant; will remain in the trial)

Participants randomised to fistula arm

1. A fistula is not usable, even after salvage procedures and several attempts

2. The fistula is usable but complication rate is high (causing dysfunction or high flow)

3. The fistula is usable but needling is not tolerated

Participants randomised to catheter arm

1. A working catheter is not achievable, even after several attempts

2. The catheter is functioning but complication rate is high

3. The catheter is functioning but not accepted

4. Drop-outs and their causes (eg, withdrawal of consent or transfer to another centre)

creation within 90 days of randomisation) with accompanying 95\% CIs will be calculated. We will describe participant characteristics and evaluate reasons for protocol violations. We will calculate rates of drop-ins and other events, although we expect low numbers. We will not conduct any interim or subgroup analyses in this pilot RCT.

\section{SAFETY DATA}

\section{Expected procedure-related events and other outcomes}

As catheter and fistula treatment strategies are standard of care in this patient population, the procedure-related outcomes are well known. We will collect only expected procedure-related events (ie, events that have a potentially causal relationship to the strategy) that occur within 7 days of the execution of any access-related intervention, as listed in table 2 . In addition, trial coordinators will track causes for hospitalisation to identify outcomes (eg, new-onset peripheral neuropathy or severe sepsis) that may have resulted from the trial procedures (box 2). Cause of death will be determined by trial site investigators, based on a trial-specific list of classifications, and will be adjudicated for accuracy by appropriate members of the trial steering committee, who will be blind to treatment arm.

\section{DISCUSSION}

An RCT comparing the effects of catheters and fistulas (the most common types of haemodialysis access being used by $>90 \%$ of patients) on clinical outcomes has never been performed. As a result, clinical decisionmaking in this area is only informed by observational studies, which are not suitable to address intervention questions due to the potential for bias. For example, data from these observational studies indicate that fistulas are superior to catheters in terms of morbidity and mortality outcomes, and healthcare costs. However, these studies compared outcomes of fistula users (typically including only people who could attain a functioning fistula) versus catheter users, including those who were not eligible to receive a fistula, those in whom a fistula was attempted but did not become usable and those who needed an access immediately available for urgent haemodialysis (fistulas require time to mature), and therefore had poorer prognosis. ${ }^{6-8}$ As a result, observational evidence indicates superior outcomes in fistula users, but does not demonstrate superiority of an access strategy based on fistulas over an access strategy based on catheters.

The questions addressed in this pilot and the hard outcome trial have been ranked among the top research priorities in dialysis by patients and healthcare providers. ${ }^{13}$ The nephrology community is increasingly aware of the limitations of available evidence. ${ }^{23}$ Nevertheless, clinical practice guidelines recommend the fistula as the ideal haemodialysis access, patient advocacy groups strongly promote fistulas over catheters and the proportion of prevalent patients using a fistula is used as a quality of care measure in some jurisdictions. ${ }^{7}$ Given the uncertainty regarding the willingness of patients and physicians to participate in an RCT in this area, a pilot RCT is necessary to prove the feasibility of a larger study.

Strengths in the design of our feasibility trial include: carefully defined feasibility objectives, clinically grounded, pragmatic approaches to enrolment (eligibility for a fistula) and to delivery of the assigned treatment (access strategy), methods to reduce bias (allocation concealment, objective outcomes, steps to ensure complete follow-up and blinding of outcome adjudicators to treatment assignment), identification of large centres with expertise in vascular access research and care and a coordinating centre with expertise in the management of large multicentre RCTs. These steps will reliably inform the design of the hard outcome trial. In addition, we have included protocols for collection of data that will be analysed in the larger study if this pilot supports feasibility (Patient-Reported Outcome Measures, vascular access questionnaire and physical function as measured by grip strength).

The design of this feasibility RCT has limitations. First, the inclusion criterion of age $\geq 65$ may limit the ability to generalise the findings to younger people who may prefer a fistula to a catheter, and in whom outcomes may differ. However, this criterion is also expected to maximise the chances of success of the pilot RCT by enhancing feasibility, and can be used to inform future studies in younger populations. Second, in the ACCESS HD trial, the question about which access strategy is best for the patient is asked after haemodialysis has started and therefore, focuses on maintaining the strategy in place (the catheter) versus changing strategy (to a fistula). Findings may not be easily generalisable to patients in whom the choice of the access is made prior to the start of haemodialysis (about 20\% of people start haemodialysis with a fistula in Canada). ${ }^{14} 15$ This design has been chosen to enhance study feasibility by limiting 
the impact of competing events (mortality before endstage kidney failure or unanticipated availability of a kidney transplant from a living donor), the possibility of changing decision about final dialysis modality or recovery of kidney function with postponement of dialysis initiation and vascular access preparation. Third, it is possible that attending nephrologists and other healthcare providers will have firm beliefs on the potential benefits of fistulas in the haemodialysis population and decline to allow their patients to be enrolled. However, a recent survey including Canadian nephrologists found that the large majority $(86 \%)$ believed that it would be ethical to randomise elderly patients into such a trial. ${ }^{23}$

The type of vascular access used for haemodialysis may have a tremendous impact on the quality and quantity of life of people receiving haemodialysis therapy. The superiority of fistulas over catheters for haemodialysis access has never been demonstrated in a randomised controlled trial, representing an important knowledge gap and research priority. The ACCESS HD pilot RCT is a vital first step to filling this critical knowledge gap and better informing practice as it will establish protocol feasibility and confirm the safety of randomly assigning patients to a catheter-based or a fistula-based strategy for haemodialysis access.

\section{TRIAL STATUS}

Registration information NCT02675569, Recruiting.

Acknowledgements The authors thank Dorothy Myers, research coordinator with the Applied Health Research Centre (AHRC) at St Michael's Hospital (Toronto, Canada), and Sharon Gulewich, study coordinator in Calgary.

Collaborator Applied Health Research Centre of St Michael's Hospital.

The ACCESS HD investigator team include: RQ: Libin Cardiovascular Institute of Alberta, Department of Medicine, and O'Brien Institute of Public Health, Department of Community Health Sciences, Cumming School of Medicine, University of Calgary; Jennifer MacRae: Libin Cardiovascular Institute of Alberta, Department of Medicine, Cumming School of Medicine, University of Calgary; Mauricio F Monroy-Cuadros: Division of Transplantation, Department of Surgery, Cumming School of Medicine, University of Calgary. Serdar Yilmaz: Division of Transplantation, Department of Surgery, Cumming School of Medicine, University of Calgary. Maria Santana: O'Brien Institute of Public Health (Department of Community Health Sciences), Cumming School of Medicine, University of Calgary; Matthew Oliver: Department of Medicine, Division of Nephrology Sunnybrook Health Science Centre, University of Toronto. Robert P Pauly: Division of Nephrology and Transplantation Immunology, University of Alberta. Sabin Shurraw: Division of Nephrology and Transplantation Immunology, University of Alberta. Lisa Miller: Max Rady College of Medicine, University of Manitoba. Clara Bohm: Section of Nephrology, Department of Medicine, Max Rady College of Medicine, University of Manitoba. Gihad Nesrallah: Li Ka Shing Knowledge Institute, St Michael's Hospital, University of Toronto. Michael Walsh: Departments of Medicine and Clinical Epidemiology and Biostatistics; Population Health Research Institute, Hamilton Health Sciences/McMaster University. Swapnil Hiremath: University of Ottawa, Faculty of Medicine and Ottawa Hospital Research Institute, Centre for Practice Changing Research. Janet Graham: The University of Ottawa, The Ottawa Hospital Research Institute. Brendan Barrett: Memorial University of Newfoundland, Patient Research Centre. Greg Browne: Clinical Chief, Division of Vascular Surgery, Eastern Health. Louise Moist: Department of Medicine, Schulich School of Medicine and Dentistry, and Kidney Clinical Research Unit, Western University. Ron Wald: Division of Nephrology, St Michael's Hospital and University of Toronto; Li Ka Shing
Knowledge Institute of St Michael's Hospital. Kevin Thorpe: Dalla Lana School of Public Health, University of Toronto, and Applied Health Research Centre of the Li Ka Shing Knowledge Institute of St Michael's Hospital. Muhammad Mamdani: Li Ka Shing Centre for Healthcare Analytics Research and Training -St Michael's Hospital, University of Toronto-Department of Medicine, University of Toronto-Leslie Dan Faculty of Pharmacy, University of Toronto -Dalla Lana Faculty of Public Health, Institute for Clinical Evaluative Sciences. PR: Libin Cardiovascular Institute of Alberta, Department of Medicine, and O'Brien Institute of Public Health, Department of Community Health Sciences, Cumming School of Medicine, University of Calgary.

Contributors $P R$ and $R Q$ conceived the study, designed the trial, obtained funding for this pilot RCT from the Canadian Institute of Health Research ( $\mathrm{CIHR}$ ) and the Kidney Foundation of Canada (KFoC) and drafted the final study protocol and the present manuscript. All listed authors (principal investigators at each site and members of the coordinating centre team) provided critical feedback to the study protocol and the present manuscript, and approved the final versions of both.

Funding University of Calgary (in kind resources); KFoC (FRN KFOC150015) and CIHR (FRN 142342) funded the ACCESS HD pilot trial.

Disclaimer Neither the study sponsor (University of Calgary) nor the funding agencies had influence on the design and set-up of the trial, or write-up of the present manuscript.

Competing interests $R Q$ is the coinventor of the Dialysis Measurement Analysis and Reporting System. Matthew Oliver is the coinventor of the Dialysis Measurement Analysis and Reporting System.

Ethics approval Conjoint Health Research Ethics Board at the University of Calgary.

Provenance and peer review Not commissioned; externally peer reviewed.

Open Access This is an Open Access article distributed in accordance with the Creative Commons Attribution Non Commercial (CC BY-NC 4.0) license, which permits others to distribute, remix, adapt, build upon this work noncommercially, and license their derivative works on different terms, provided the original work is properly cited and the use is non-commercial. See: http:// creativecommons.org/licenses/by-nc/4.0/

\section{REFERENCES}

1. Liyanage $T$, Ninomiya $T$, Jha $V$, et al. Worldwide access to treatment for end-stage kidney disease: a systematic review. Lancet 2015;385:1975-82.

2. United States Renal Data System. Annual Data Report 2015. http:// www.usrds.org/ (accessed 2 Jun 2016).

3. Thomas B, Wulf S, Bikbov B, et al. Maintenance dialysis throughout the world in years 1990 and 2010. J Am Soc Nephrol 2015;26: 2621-33.

4. Jindal K, Chan CT, Deziel C, et al. Hemodialysis clinical practice guidelines for the Canadian Society of Nephrology. J Am Soc Nephrol 2006;17(3 Suppl 1):S1-27.

5. Tordoir J, Canaud B, Haage P, et al. EBPG on vascular access. Nephrol Dial Transplant 2007;22(Suppl 2):ii88-117.

6. Ravani P, Palmer SC, Oliver MJ, et al. Associations between hemodialysis access type and clinical outcomes: a systematic review. J Am Soc Nephrol 2013;24:465-73.

7. Quinn RR, Ravani P. Fistula-first and catheter-last: fading certainties and growing doubts. Nephrol Dial Transplant 2014;29:727-30.

8. Tennankore KK, Soroka SD, Kiberd BA. The impact of an 'acute dialysis start' on the mortality attributed to the use of central venous catheters: a retrospective cohort study. BMC Nephrol 2012;13:72.

9. Brown RS, Patibandla BK, Goldfarb-Rumyantzev AS. The survival benefit of 'Fistula First, Catheter Last' in hemodialysis is primarily due to patient factors. J Am Soc Nephrol Published Online First: 7 Sep 2016. doi: 10.1681/ASN.2016010019.

10. Quinn RR, Oliver MJ, Devoe D, et al. The effect of predialysis fistula attempt on risk of all-cause and access-related death. J Am Soc Nephrol Published Online First: 6 Oct 2016. doi:10.1681/ ASN.2016020151.

11. Casey JR, Hanson CS, Winkelmayer WC, et al. Patients' perspectives on hemodialysis vascular access: a systematic review of qualitative studies. Am J Kidney Dis 2014;64:937-53. http://dx. doi.org/10.1053/j.ajkd.2014.06.024 
12. Lee $\mathrm{H}$, Manns $\mathrm{B}$, Taub K, et al. Cost analysis of ongoing care of patients with end-stage renal disease: the impact of dialysis modality and dialysis access. Am J Kidney Dis 2002;40:611-22.

13. Manns B, Hemmelgarn B, Lillie E, et al. Setting research priorities for patients on or nearing dialysis. Clin J Am Soc Nephrol 2014;9:1813-21.

14. Zhang JC, Al-Jaishi AA, Na Y, et al. Association between vascular access type and patient mortality among elderly patients on hemodialysis in Canada. Hemodial Int 2014;18:616-24.

15. Robinson BM, Akizawa T, Jager KJ, et al. Factors affecting outcomes in patients reaching end-stage kidney disease worldwide: differences in access to renal replacement therapy, modality use, and haemodialysis practices. Lancet 2016;388:294-306.

16. Hicks CW, Canner JK, Arhuidese I, et al. Mortality benefits of different hemodialysis access types are age dependent. J Vasc Surg 2015;61:449-56.

17. Lok CE, Allon M, Moist $\mathrm{L}$, et al. Risk equation determining unsuccessful cannulation events and failure to maturation in arteriovenous fistulas (REDUCE FTM I). J Am Soc Nephrol 2006;17:3204-12.
18. Murea M, James KM, Russell GB, et al. Risk of catheter-related bloodstream infection in elderly patients on hemodialysis. Clin J Am Soc Nephrol 2014;9:764-70.

19. Quinn RR, Lamping DL, Lok CE, et al. The Vascular Access Questionnaire: assessing patient-reported views of vascular access. $J$ Vasc Access 2008;9:122-8.

20. Chan AW, Tetzlaff JM, Altman DG, et al. SPIRIT 2013 statement: defining standard protocol items for clinical trials. Ann Intern Med 2013;158:200-7.

21. Oliver MJ, Verrelli M, Zacharias JM, et al. Choosing peritoneal dialysis reduces the risk of invasive access interventions. Nephrol Dial Transplant 2012;27:810-16.

22. Kosa SD, Al-Jaishi AA, Moist L, et al. Preoperative vascular access evaluation for haemodialysis patients. Cochrane Database Syst Rev 2015;(9):CD007013.

23. Poinen $\mathrm{K}$, Oliver MJ, Ravani $\mathrm{P}$, et al. Willingness to participate in a randomized trial comparing catheters to fistulas for vascular access in incident hemodialysis patients: an international survey of nephrologists. Can J Kidney Health Dis. Published Online First: 13 Jul 2016. doi:10.1186/s40697-016-0125-6. 